\title{
Chronic electrocorticography for sensing movement intention and closed-loop deep brain stimulation with wearable sensors in an essential tremor patient
}

\author{
Jeffrey A. Herron, PhD, ${ }^{1,5}$ Margaret C. Thompson, MS, ${ }^{1,5}$ Timothy Brown, MA, ${ }^{2,5}$ \\ Howard J. Chizeck, ScD, ${ }^{1,3,5}$ Jeffrey G. Ojemann, MD, ${ }^{4,5}$ and Andrew L. Ko, MD ${ }^{4,5}$
}

Departments of ${ }^{1}$ Electrical Engineering, ${ }^{2}$ Philosophy, ${ }^{3}$ Bioengineering, and ${ }^{4}$ Neurological Surgery, University of Washington; and ${ }^{5}$ NSF Engineering Research Center for Sensorimotor Neural Engineering, Seattle, Washington

\begin{abstract}
Deep brain stimulation (DBS) has become a widespread and valuable treatment for patients with movement disorders such as essential tremor (ET). However, current DBS treatment constantly delivers stimulation in an open loop, which can be inefficient. Closing the loop with sensors to provide feedback may increase power efficiency and reduce side effects for patients. New implantable neuromodulation platforms, such as the Medtronic Activa PC+S DBS system, offer important data sources by providing chronic neural sensing capabilities and a means of investigating dynamic stimulation based on symptom measurements. The authors implanted in a single patient with ET an Activa PC+S system, a cortical strip of electrodes on the hand sensorimotor cortex, and therapeutic electrodes in the ventral intermediate nucleus of the thalamus. In this paper they describe the effectiveness of the platform when sensing cortical movement intentions while the patient actually performed and imagined performing movements. Additionally, they demonstrate dynamic closed-loop DBS based on several wearable sensor measurements of tremor intensity.
\end{abstract}

https://thejns.org/doi/abs/10.3171/2016.8.JNS16536

KEY WORDS deep brain stimulation; essential tremor; ECoG; closed-loop systems; functional neurosurgery

$\mathrm{D}$ EEP brain stimulation (DBS) has proven to be an important tool in treating symptoms of medication-resistant neurological disorders. Modern DBS implants treat essential tremor (ET), Parkinson's disease (PD), and dystonia by constantly delivering electrical pulses to neural tissue in subcortical structures. ${ }^{11}$ However, current treatment with DBS leaves much to be desired in terms of device longevity and unwanted side effects. The systems must be surgically replaced when the battery is depleted, and stimulation can cause paresthesias, dysarthria, and capsular and cognitive side effects. ${ }^{8}$ Additionally, the neurological disorders that DBS treats are highly variable day to day or even moment to moment. For instance, many patients with ET only experience tremor while performing intentional movement with their affected limb, with no noticeable tremor at rest. ${ }^{1}$ Such a patient only benefits from the stimulation while moving his or her limb but suffers any side effects of stimulation, all while the device is constantly draining the battery regardless of whether the patient is moving a limb or not. Currently, adjustments to DBS are performed in an "open-loop" fashion, in which changes to stimulation parameters are made by a clinician or, in a limited fashion, by the patient, using an external device.

There is ongoing work to address these shortcomings by developing a "closed-loop" system in which sensors are used to estimate symptom intensity and adjust stimulation accordingly without direct physical intervention by a clinician or the patient. ${ }^{4}$ These closed-loop DBS (CLDBS) systems may allow stimulation to be delivered only when needed, at just the right intensity to treat symptoms. Testing such systems in humans has begun, with wearable sensors being used to modify stimulation based on tremor measurements for both ET patients ${ }^{14}$ and PD patients. ${ }^{9}$

For ET patients who experience tremor during intentional movement, stimulation changes could be triggered

ABBREVIATIONS CLDBS = closed-loop DBS; DBS = deep brain stimulation; ECoG = electrocorticography; EMG = electromyography; ET = essential tremor; IMU = inertial measuring unit; $\mathrm{PD}=$ Parkinson disease; $\mathrm{PSD}=$ power spectral density; TRS = Fahn-Tolosa-Marin Tremor Rating Scale; VIM = ventral intermediate nucleus of the thalamus.

SUBMITTED March 8, 2016. ACCEPTED August 8, 2016.

INCLUDE WHEN CITING Published online November 18, 2016; DOI: 10.3171/2016.8.JNS16536. 
based on sensors that either detect tremor or identify volitional movement. Wearable inertial or electromyography (EMG) sensors are both attractive options because of their ability to directly monitor a patient's limb movements. ${ }^{5}$ Another potential signal source to determine when a patient will experience tremor is the motor cortex. ${ }^{6}$ Betaband desynchronization in the motor cortex has long been known to be associated with intentional movement of the limb, and these signals can be clearly observed using either electroencephalography on the scalp ${ }^{12}$ or electrocorticography $(\mathrm{ECoG})$ on the brain surface. ${ }^{3}$ Using either type of signal may permit systems to automatically determine when and how stimulation is needed, allowing the patient to experience side effects only while actually using his or her arm. Additionally, such systems may allow increased device battery life given the reduced amount of stimulation output.

To investigate the utility of both cortical neural sensing and dynamic stimulation adjustment in patients with ET, we implanted in a single ET patient a stimulation lead in the ventral intermediate nucleus of the thalamus (VIM) and a subdural electrode strip over the patient's motor cortex, connected to an Activa PC+S DBS generator. The Activa $\mathrm{PC}+\mathrm{S}$ system is a Medtronic neurostimulator that can sense data from implanted electrodes. ${ }^{10}$ The sensed data can be streamed to an external computer onto which they can be logged, control external devices, or trigger stimulation changes. In the following case report, we describe the patient's clinical data, his surgical outcomes, and the results from several preliminary experimental sessions with him. We demonstrate the system's sensing capability during movement tasks overtly performed and imagined. Using wearable inertial and EMG sensors on the tremoraffected arm, we then demonstrate the ability to deliver responsive closed-loop dynamic stimulation to treat tremor in a closed-loop fashion.

\section{Case Report}

History and Examination

A 58-year-old right-handed man presented to his neurologist with an action tremor noted for about 4 years. This tremor affected mainly his right upper and lower extremities with minimal left-sided symptoms and no head or voice tremor. Tremor was not present at rest and worsened with intentional movement. The patient did not have any history suggestive of rapid eye movement (REM) sleep behavior disorder, constipation, postural dizziness, or gait or balance problems. He had had no exposure to dopamine antagonists. His tremor became more severe over 2 years, which significantly impacted his activities of daily life, particularly when eating and drinking. The patient did not drink alcohol and did not notice that alcohol improved his tremor. His family history was unknown as he was adopted. Propranolol did not have significant benefit, and a trial of primidone was not tolerated because of side effects.

On neurological examination, his affect and speech were normal. He scored 29/30 on the Montreal Cognitive Assessment (MoCA). He had no hypomimia or hypophonia. Cranial nerves II-XII were intact. He had no motor or sensory deficits. He had no ataxia. Rapid alternating movements were normal and symmetric. Gait and arm swing were normal; he rose from a chair briskly without using his arms. He independently recovered with 1 step on the retropulsive pull test.

\section{Presurgical Evaluation}

The patient had no head or voice tremor and no tremor at rest. His tremor on the right side was evaluated to be severe during action and while holding posture. On the left side, there was minimal to no tremor. The Fahn-TolosaMarin Tremor Rating Scale (TRS) was used to evaluate tremor severity preoperatively (scores: Part A, 13; Part B, 10; Part C, 10). Preoperative gait and balance evaluations were performed, with no fall risk identified on the Berg Balance Test (55/56), 10-Meter Walk Test $(1.6 \mathrm{~m} /$ sec), and Dynamic Gait Index (22/24). Preoperative and perioperative imaging revealed no concerning anatomical lesions. A DaTscan (GE Healthcare) showed no evidence of nigrostriatal degeneration. Magnetic resonance images obtained for operative planning included a 3-T volumetric $\mathrm{T} 1$ magnetization-prepared rapid acquisition gradient echo (MPRAGE) sequence (1.5-mm slice thickness, $512 \times$ 512 matrix, FOV 250).

\section{Operative Procedure}

Surgical treatment options were discussed with the patient, and informed consent for implantation of the DBS system targeting the left VIM was obtained. Separately, in accordance with institutional review board and Investigational Device Exemption regulations, research staff approached the patient, and he was enrolled in this study.

The patient was brought to the operating room and intravenous sedation was initiated. He was positioned supine, and his head was placed in 3-point fixation using a skull clamp and long Doro Transitional Member Radiolucent headrest system (Pro Med Instruments $\mathrm{GmbH}$ ) after infiltration of the pin sites with a local anesthetic. Bone fiducials (Medtronic Inc.) were affixed to the skull, and an intraoperative CT scan (1.25-mm slice spacing, $512 \times 512$ matrix, FOV 250) was obtained using the CereTom scanner (NeuroLogica Inc.). The CT and MRI studies were merged using FrameLink (Medtronic Inc.), and an operative plan targeting the left VIM was created. The hand motor cortex was identified anatomically, and scalp overlying this target was marked in indelible marker.

Electrode placement was performed using the NexFrame frameless stereotactic system (Medtronic Inc.), as previously described, ${ }^{2}$ with the following modifications. After securing the Stimloc bur hole cover (Medtronic Inc.) to the skull and before placement of the NexFrame base, the dura mater was opened widely in a cruciate fashion. A Resume II electrode (model 3587, Medtronic Inc.) was introduced into the subdural space directed toward the hand motor cortex. The hand motor cortex was localized using anatomical landmarks on MRI. The central sulcus was located using axial and sagittal views. The cortical electrode was manipulated to straddle the central sulcus at the level of the "hand knob" on MRI, and additional CT scanning was performed to confirm this localization. The spacing of the distal contacts on the Resume II electrode is not compatible with our standard cables for testing somatosensory 

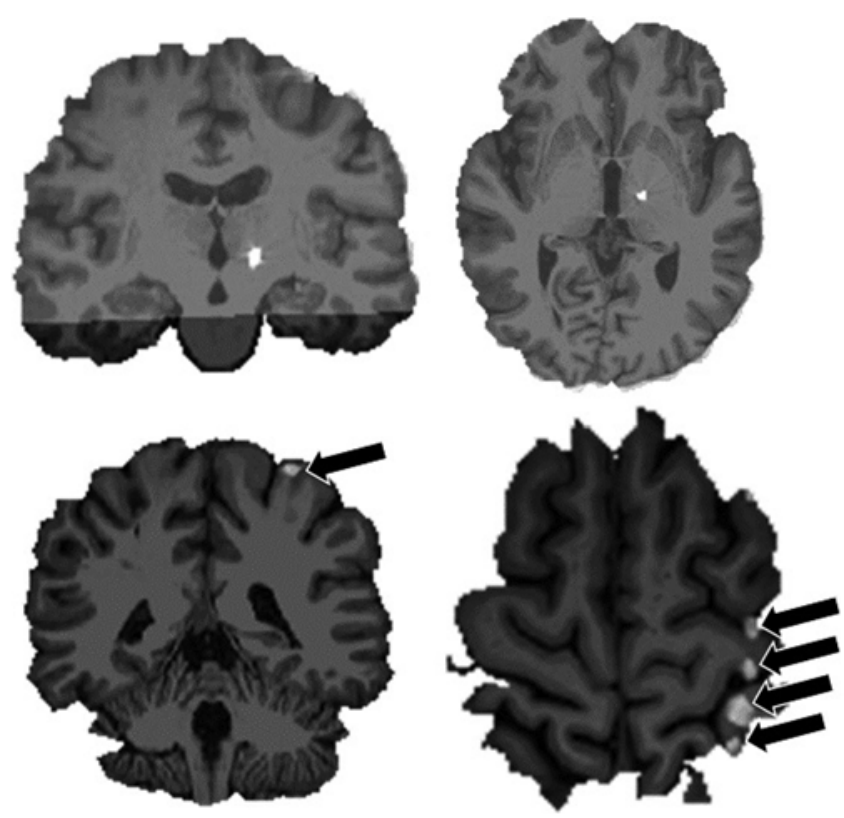

FIG. 1. Postoperative imaging confirmed VIM lead and cortical electrode placement. Upper: Coronal and axial views of merged T1-weighted MR image and intraoperative CT scan showing target electrode (Contact 1) within the thalamus. Lower: Coronal and axial views of merged T1-weighted MR image and intraoperative CT scan showing location of cortical electrodes. Arrows denote locations of cortical electrodes overlying sensorimotor cortex.

or motor evoked potentials, and this testing was not performed. A channel was drilled into the Stimloc bur hole cover for egress of this electrode. The thalamic lead (model 3387, Medtronic Inc.) was then placed at the target. An intraoperative CT was obtained to confirm the location of the thalamic electrode and cortical strip. Vector error to target was $0.3 \mathrm{~mm}$ for the thalamic electrode. Cortical electrodes were adjusted such that 2 contacts were situated over presumed hand motor cortex and 2 were over hand sensory cortex. The final locations of the implanted electrodes for cortical sensing and VIM stimulation are shown in Fig. 1.

The patient was allowed to awaken for macroelectrode stimulation testing. He exhibited a robust microlesion effect with nearly complete extinction of tremor. Testing for side effects with bipolar stimulation (Contact $0-3+$, pulse width 90 usec, rate $130 \mathrm{~Hz}$ ) showed none up to an amplitude of $4.0 \mathrm{~V}$, when transient paresthesias were noted. At $5.0 \mathrm{~V}$, the patient exhibited mild dysarthria. He was reanesthetized, electrodes were secured, and closure of soft tissues proceeded in a standard fashion.

One week later, the patient underwent implantation of the Activa $\mathrm{PC}+\mathrm{S}$ generator (Medtronic Inc.) while under general anesthesia, without complication. This is an experimental device with limited availability from Medtronic under an Investigational Device Exemption from the Food and Drug Administration. Externally, it is identical to the dual-lead Activa PC. However, this device has the capability to acquire electrophysiological data as well as provide clinical DBS. It is programmable with the Medtronic 8840 clinical programmer but has the capability to store limited amounts of electrophysiological data, as well as stream

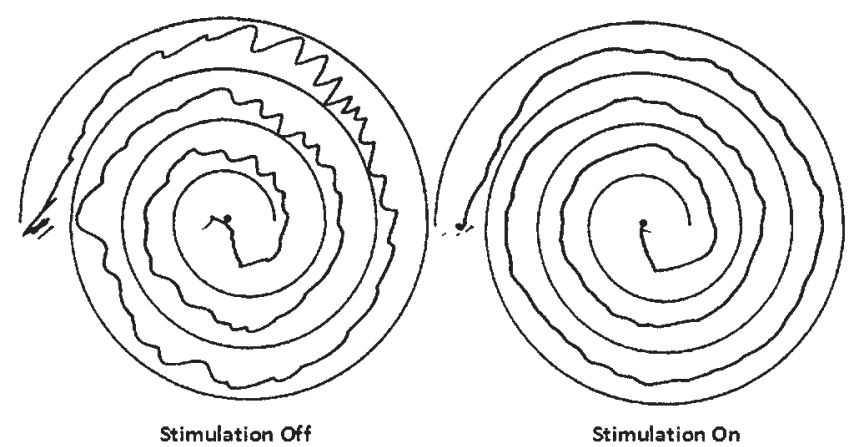

FIG. 2. The patient's spiral drawing with stimulation on (right) and off (left), obtained during a tremor assessment before the experiments.

these data in real time via the Nexus-D (Medtronic Inc.), which allows bidirectional communication between the $\mathrm{PC}+\mathrm{S}$ pulse generator and a computing system via Universal Serial Bus (USB) connection.

\section{Postoperative Course}

At the patient's first postoperative visit 2 weeks after generator implantation, he continued to show a microlesion effect but with minimal return of tremor. Monopolar mapping was performed, with no lasting side effects seen with stimulation up to $4.0 \mathrm{~V}$ (pulse width $90 \mu \mathrm{sec}$, rate 130 $\mathrm{Hz}$ ). Six weeks after surgery, the device was programmed with the following settings: electrode configuration $2+1-$, pulse width $90 \mu \mathrm{sec}$, rate $130 \mathrm{~Hz}$, amplitude $3.7 \mathrm{~V}$. When he returned 10 weeks postoperatively, he noted transient paresthesias when the system was turned on and off. The device was reprogrammed to his current settings, with an electrode configuration of $2+1-$, pulse width $90 \mu \mathrm{sec}$, rate $150 \mathrm{~Hz}$, and amplitude $2.5 \mathrm{~V}$. His TRS scores with stimulation have greatly improved (Part A: 1, Part B: 3). Figure 2 illustrates the benefit this patient receives from DBS during a standard spiral drawing task. As can be seen, there is a marked difference in his ability to draw spirals between the stimulation off and on states.

\section{Sensing of Actual and Imagined Movements}

To characterize signals recorded from the cortical electrodes during periods of movement and intention, we designed a task in which a computer prompted the user to move his arm or hand or to rest while we measured the limb activity using a worn accelerometer. We prompted the patient to alternate between movement and rest every 3 seconds to collect 20 trials each of arm and hand movements and 40 trials of rest. The patient was then asked to only imagine the movements of the same tasks, and the worn accelerometer was used to confirm that they did not accidentally perform actual movements. During both trials the Medtronic Nexus-D system was used to stream data from the Activa $\mathrm{PC}+\mathrm{S}$ to a laptop at which it was time-stamped and logged. The Activa $\mathrm{PC}+\mathrm{S}$ sensing lead was configured to record data sensed differentially from the hand M1 to S1 cortex sampled at $422 \mathrm{~Hz}$. It is important to note that the patient did not experience tremor while performing any of the prompted movements. Example time-series plots of the raw data collected from these 

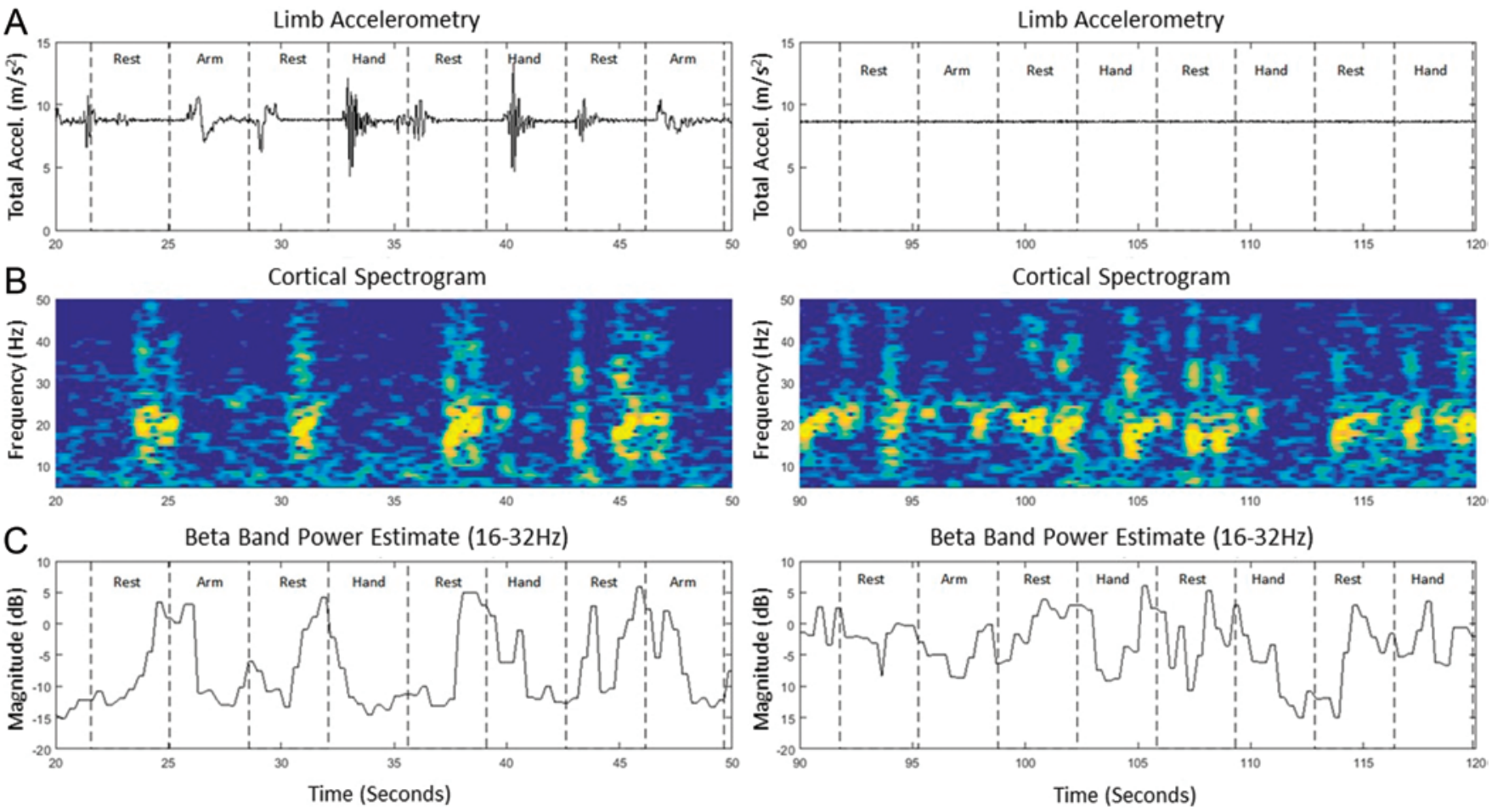

FIG. 3. Example segments from experimental trials during which the patient performed prompted movements for the arm and hand or rested. Actual-movement trials appear on the left, and imagined-movement trials appear on the right. The nature of the prompted movements for each 3-second interval is indicated by text. A: Limb accelerometry captured from a wrist-worn inertial sensor. B: Spectrograms of streamed ECoG data from M1 to S1 electrodes. C: Real-time cortical beta-band power.

trials are displayed in Fig. 3 for both actual and imagined movement experiments.

We then segmented the data into periods based on the prompted action. The power spectral density (PSD) was calculated for each prompted action using Welch's method with a 512-sample-long Hann window with $80 \%$ overlap. The output PSD was then averaged with the other trials with the same prompted action to generate an average PSD for a given state. These average PSD plots are displayed in Fig. 4 for both the overt and imagined movement tasks. Since the patient often took some time to react to the prompt and change his limb's state, the PSDs excluded the 1 st second of every prompted action.

As can be seen in the overt movement PSD plots in Fig. 4, the cortical signal's beta band is desynchronizing as expected whenever the patient moves his limb. During rest, the patient seems to have beta peaks at both 20 and $32 \mathrm{~Hz}$. The beta-band power during overt arm and hand movements illustrates a broad drop in band power across the entire band, with a more pronounced beta-band desynchronization occurring during hand movement. However, for imagined movement, only a small band around the $32-\mathrm{Hz}$ peak lowered on average during the prompted imaginary actions, but the results were so variable that it was difficult to distinguish between resting and imagined movement.

\section{Closed-Loop DBS With Wearable Inertial and EMG Sensors}

To demonstrate CLDBS using this system, we asked the patient to move his hand from his lap and to hold it in front of his nose. This task consistently evoked tremor in the stimulation-off state. We collected inertial and EMG data from the shaking limb while he performed this action and used the sensed data to trigger stimulation changes in real time. The inertial measuring unit (IMU) we used was an Android smartwatch with a custom application sampling the on-board accelerometer and gyroscope at 100 Hz. The EMG system used was a gTec Mobilab, which uses active electrodes and samples data at a $256-\mathrm{Hz}$ rate. For the first system, we used the inertial sensor to deliver stimulation based on the presence of tremor. The system would increase or decrease stimulation based on the magnitude of the tremor band power between 4 and $8 \mathrm{~Hz}$ on the 3 gyroscope channels. This band was chosen because the patient's primary tremor frequency was around $5 \mathrm{~Hz}$. For the second system, we used EMG to trigger stimulation changes whenever the patient volitionally moved his limb. To accomplish this we attached 4 EMG electrodes to the patient's arm (bicep, triceps, anterior forearm, posterior forearm) and triggered stimulation whenever the total EMG band power $(4-50 \mathrm{~Hz})$ summed to a value larger than a calibrated threshold. The experimental results of the patient's responses while using these systems are shown in Fig. 5.

The plot is separated into 4 distinct system states: no stimulation, open-loop stimulation, IMU tremor-modulated stimulation, and EMG movement-triggered stimulation. As can be seen in the gyroscope magnitude plot, there are large peaks whenever the patient started or stopped movement, which carries over into the $4-$ to $8-\mathrm{Hz}$ band power 

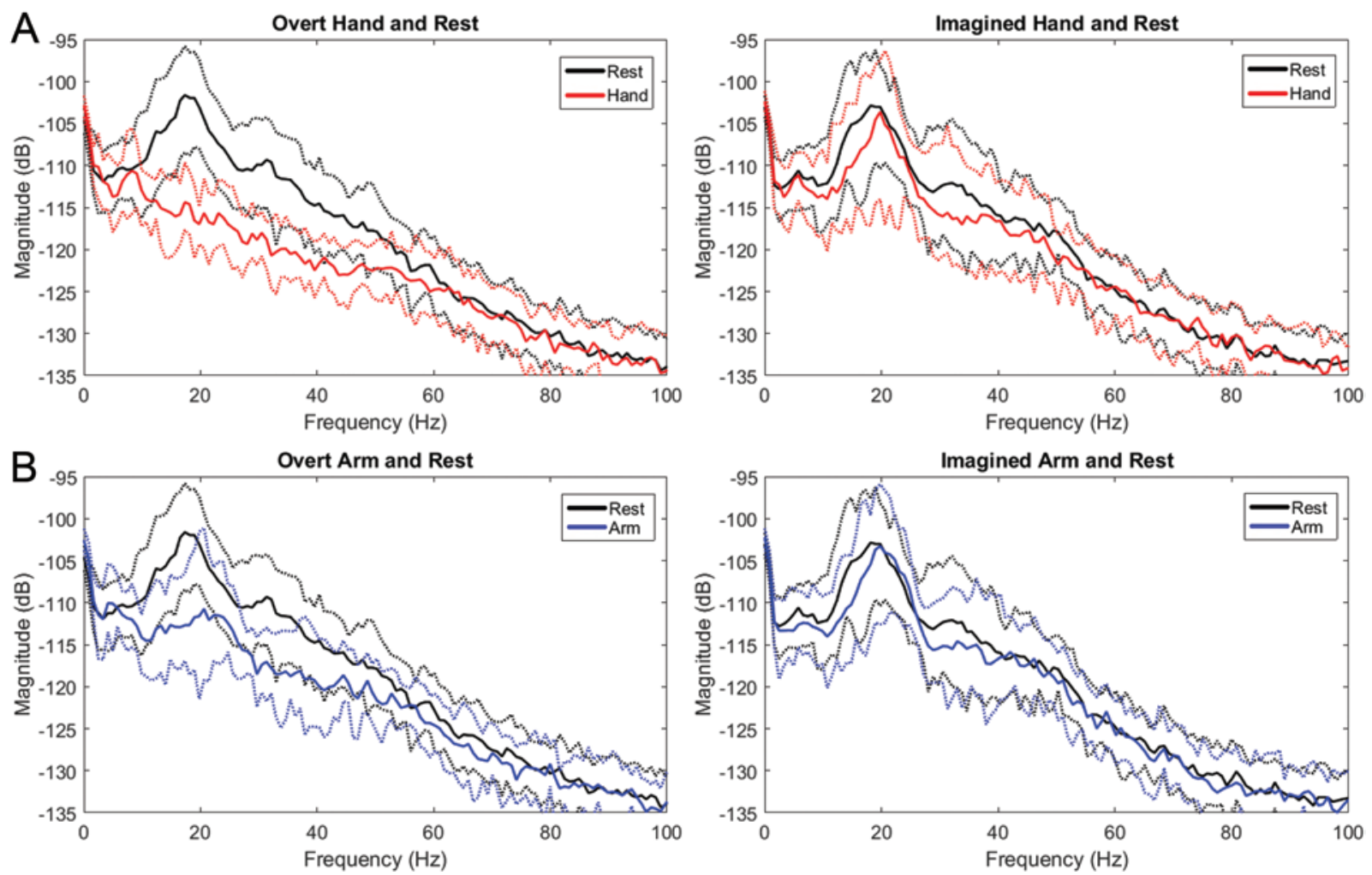

FIG. 4. Mean power spectral density (PSD) plot for each of the 3 prompted actions for both overt (left) and imagined (right) movement experiments. Because of patient reaction times, the PSD was calculated based on the 1- to 3-second time period after each prompt, to remove state transitions. The PSD mean and quantile bounds were then calculated based on results from all prompts of a specific type. Solid lines indicate the mean PSD, and dotted lines indicate the 0.9 to 0.1 quantile bounds. Note the drop in average beta-band power during overt arm (B) or hand $(\mathbf{A})$ movement.

plot. These artifacts are caused by the cross-band frequency components of initiating movement and the arm impacting the lap when the task was complete. To remove these artifacts and determine a quantified estimate of the actual tremor the patient was experiencing, we multiplied the tremor band power by the proportion of the tremor band over the total band power. This takes advantage of the large cross-band nature of the artifact and results in good removal of the initiation and impact events, as can be seen in the tremor intensity plot.

With a quantified estimate of the tremor throughout the trial, we can assess the amount of tremor the patient experiences while performing the limb movement in each of the 4 states. The quantifiable metrics of tremor and power usage during the open-loop, tremor-modulated, and movement-triggered trials are compiled in Table 1. The tremor experienced by the patient in each state is normalized to the open-loop tremor. The stimulation power difference is determined by comparing the closed-loop stimulation power with the open-loop settings. The final column represents the trade-off between power and any additional tremor the patient would experience while using the closed-loop system as compared with the open-loop version. The rationale and methods for these CLDBS metrics are discussed in a separate paper. ${ }^{7}$ While using the EMGbased movement-sensing system, the patient experienced an additional $8.2 \%$ of tremor while decreasing power us- age by $53 \%$, which is a $6.5 \%$ gain in power efficiency for every $1 \%$ of additional tremor. With the tremor-modulated system, the patient experienced much higher power savings of $84.5 \%$ at the cost of significant (36.2\%) additional tremor, resulting in a $2.3 \%$ gain in power efficiency for every $1 \%$ increase in tremor.

\section{Discussion}

This case study demonstrates that the Activa $\mathrm{PC}+\mathrm{S}$ system with cortical electrodes is a robust, fully implantable platform to research improving neuromodulation technologies for patients with movement disorders. Implanting both VIM and cortical electrodes in an ET patient opens up the ability to investigate the use of motor cortex signals for the purposes of creating a CLDBS system. We have begun our preliminary research using this system to sense movement intentions and have performed exploratory CLDBS experiments using wearable sensors to provide symptom feedback.

The results from our movement-prompting task, shown in Figs. 3 and 4, show that overt movements are clearly distinguishable from rest given a broad movement-related beta-band desynchronization. ${ }^{9}$ These beta-band changes correlate well temporally with inertial and EMG evidence of movement. However, our patient's imagined movements are much harder to distinguish, with a smaller desynchro- 


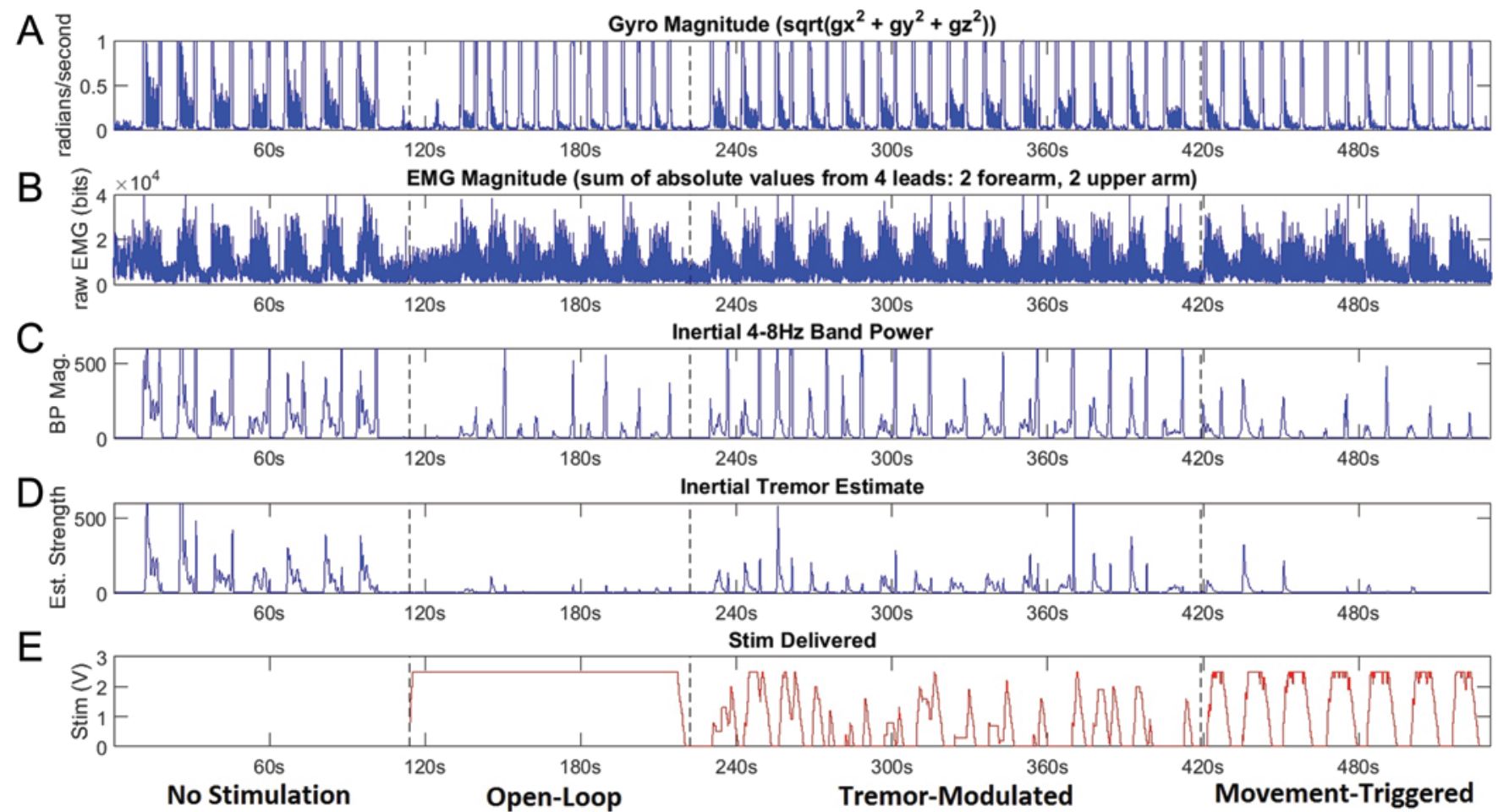

FIG. 5. Time domain plots of experimental CLDBS trial. A: Gyroscope (Gyro) magnitude plot over the duration of the trial. Large peaks are movement initiation and impact events. sqrt = square root. B: Magnitude of the raw EMG recorded through the trial, summed across 4 leads on the upper limb. C: Gyroscope band power in the 4- to 8-Hz frequency range. Note that movement initiation and impact artifacts are still present. BP Mag. = band power magnitude. D: Tremor intensity estimate (Est.) taken by weighting the tremor band power by the proportion of the tremor band power over the total band power. The tracings are still representative of tremor but with smaller effects of initiation and impact. E: Stimulation (Stim) delivered to the patient in each of the 4 states.

nization only noticeable between 26 and $36 \mathrm{~Hz}$. This patient's beta band does not have the broad desynchronization during imagined movement, which has been seen in other literature. ${ }^{13}$ The reason for this finding is unclear but possibly arises from patient difficulty in imagining movement.

The results from our preliminary CLDBS trials indicate that the patient's tremor can be reduced while receiving selective and dynamic stimulation. For this patient and these algorithms, the EMG-based movement-triggered system performed better during these trials than the IMU tremor-modulated system. This may be due to the inertial system's requirement that tremor be present to respond. Thus, it is expected that there will be additional tremor when compared with the open-loop or EMG movementtriggered case. For the EMG movement-triggering system, it is important to note that the patient can make movements that do not result in tremor but that the system will still trigger stimulation. Thus, the EMG movement-triggering algorithm can result in unneeded stimulation when used outside of the clinic. However, this may still provide a large enough reduction in stimulation power to be of benefit. Future work will examine the prediction of tremor and determining the optimal amount of stimulation to treat it in real time.

There are multiple considerations when moving forward from the paradigm described to a neural-triggered closed-loop system. For example, the external sensors we used have very little latency, with near instantaneous access to EMG and inertial sensing data, with high bandwidth rates and high sampling rates. Using the embedded sensing capabilities of the $\mathrm{PC}+\mathrm{S}$ system will introduce latency given the need for communication with the device; likewise, limits in the bandwidth of such communication will impose conditions on the sampling rate. For example, because of hardware limitations, exploring known cortical markers in the gamma band $(>65 \mathrm{~Hz})$ was not feasible during these experiments. How such considerations will impact the development of algorithms and control systems is difficult to predict. A simple threshold in total beta-power decrease over time, for instance, would be a straightforward method to trigger stimulation; alternatively, examination of higher-order interactions between electrodes may prove a more accurate but more computationally costly implementation. A forthcoming firmware update for the current system will allow for better telemetry and will likely make it possible to use the gamma band as a potential cortical marker. Importantly, the consideration of accuracy in stimulation should take into account that an algorithm with a low false-negative and a potentially high false-positive rate (that is, delivery of stimulation when needed and sometimes when not needed) is very likely to be superior to the current paradigm, in which the stimulator is on $100 \%$ of the time. 
TABLE 1. Quantified CLDBS results

\begin{tabular}{|c|c|c|c|c|c|c|c|}
\hline \multirow[b]{2}{*}{$\begin{array}{l}\text { Control } \\
\text { Mode }\end{array}$} & \multicolumn{2}{|c|}{ Open-Loop System } & \multicolumn{2}{|c|}{ Closed-Loop System } & \multicolumn{3}{|c|}{ CLDBS Metrics } \\
\hline & $\begin{array}{l}\text { Stimulation } \\
\text { Amplitude }\end{array}$ & $\begin{array}{l}\text { Untreated } \\
\text { Tremor† }\end{array}$ & $\begin{array}{l}\text { Average Stimulation } \\
\text { Amplitude }\end{array}$ & $\begin{array}{l}\text { Untreated } \\
\text { Tremor† }\end{array}$ & $\begin{array}{c}\text { Power } \\
\text { Reduction }\end{array}$ & $\begin{array}{c}\text { Tremor } \\
\text { Difference§ }\end{array}$ & $\begin{array}{c}\text { Stimulation-Tremor } \\
\text { Trade-Off } 斤\end{array}$ \\
\hline Tremor & $2.5 \mathrm{~V}$ & $5.3 \%$ & $0.7 \mathrm{~V}$ & $41.6 \%$ & $84.5 \%$ & $36.2 \%$ & $2.3 \%$ \\
\hline Movement & $2.5 \mathrm{~V}$ & $5.3 \%$ & $1.4 \mathrm{~V}$ & $13.5 \%$ & $53.2 \%$ & $8.2 \%$ & $6.5 \%$ \\
\hline
\end{tabular}

* Determined by taking the average stimulation amplitude over the entire time duration in a given state.

$\dagger$ Values represent the percentage of the no-stimulation tremor that the system cannot suppress. They are determined by dividing the average tremor band power in each mode by the average tremor band power with no stimulation.

$\ddagger$ Represents the average stimulation power savings that a closed-loop system provides over the open-loop case.

$\S$ Represents the increased tremor that the closed-loop system fails to suppress compared to the open-loop system and normalized to the no-stimulation case.

I The proportional trade-off between stimulation power savings and increased tremor for the closed-loop system.

It is also worth considering the patient's perspective on the possibility of CLDBS and the need for power conservation. During conversations with him across several postoperative research visits, the patient was very interested in conserving battery power for multiple reasons. At his first research visit, he was taken aback by how much the neurostimulator protruded from his chest; he wondered if a system that conserves battery power could be built with a smaller battery and, thus, a thinner profile. At subsequent visits, he noted that he keeps his stimulator turned off $95 \%$ of the time: he only turns it on so that he can eat without tremor symptoms. When asked why he turns his stimulator off, he explained that he wanted to conserve his battery in the hopes of avoiding follow-up surgery. A closed-loop system would make it possible for the patient to conserve battery power without manual intervention, potentially allowing for longer battery life and fewer battery replacement surgeries. These conversations suggest that saving battery power is worth some additional tremor for some patients. Perhaps future closed-loop systems will not need to reach the same level of tremor reduction as an openloop system before being an attractive treatment option for some individuals.

We are now combining these 2 preliminary investigations to develop ECoG-triggered CLDBS systems. In this case, the observed beta-band desynchronization during movement could be used to trigger stimulation changes in the patient. This would be similar to how we have used EMG to trigger stimulation on movement, with the possible advantage that an ECoG-triggered system is fully implantable without the need to communicate with external components. This would further increase the power efficiency of future CLDBS paradigms given the fact that transmitting across the skin barrier is incredibly power intensive for an implanted device.

\section{Conclusions}

This case report covers the first steps for developing novel fully implanted systems that use cortical signals for therapeutic applications. Our data clearly indicate that we can use cortical signals to identify periods of movement for future movement-triggered CLDBS trials. Additionally, we have demonstrated the ability to close the loop to provide real-time stimulation adjustment based on worn sensors. This work lays the foundation for future investigations into the development of novel bidirectional neural prosthetics including neural-triggered stimulation to allow for fully implantable CLDBS systems that can make moment-to-moment decisions on how and when to stimulate for tremor. The future research prospects with this patient and implanted systems are exciting, and this case report will help to pave the way for more bidirectional neural prosthetics work in the future to aid in the treatment of ET and other movement disorders.

\section{Acknowledgments}

This work was supported by Award No. EEC-1028725 from the National Science Foundation for the Center for Sensorimotor Neural Engineering. The content is solely the responsibility of the authors and does not necessarily represent the official views of the National Science Foundation. This research was also supported by the Department of Defense (DoD) through the National Defense Science and Engineering Graduate Fellowship (NDSEG) Program.

\section{References}

1. Benito-León J, Louis ED: Clinical update: diagnosis and treatment of essential tremor. Lancet 369:1152-1154, 2007 (Erratum in Lancet 370:566, 2007)

2. Burchiel KJ, McCartney S, Lee A, Raslan AM: Accuracy of deep brain stimulation electrode placement using intraoperative computed tomography without microelectrode recording. J Neurosurg 119:301-306, 2013

3. Crowell AL, Ryapolova-Webb ES, Ostrem JL, Galifianakis NB, Shimamoto S, Lim DA, et al: Oscillations in sensorimotor cortex in movement disorders: an electrocorticography study. Brain 135:615-630, 2012

4. Hebb AO, Zhang JJ, Mahoor MH, Tsiokos C, Matlack C, Chizeck HJ, et al: Creating the feedback loop: closed-loop neurostimulation. Neurosurg Clin N Am 25:187-204, 2014

5. Herron J, Chizeck HJ: Prototype closed-loop deep brain stimulation systems inspired by Norbert Wiener, in 2014 IEEE Conference on Norbert Wiener in the 21st Century (21CW). New York: IEEE, 2014 (http://brl.ee. washington.edu/wp-content/uploads/2014/06/Herron_ ClosedLoopDBSPrototypes.pdf) [Accessed September 20, 2016]

6. Herron J, Denison T, Chizeck HJ: Closed-loop DBS with movement intention, in 7th International IEEE/EMBS Conference on Neural Engineering. New York: IEEE, 2015, pp 844-847 (http://ieeexplore.ieee.org/lpdocs/epic03/wrapper. htm?arnumber=7146755) [Accessed September 20, 2016]

7. Herron J, Velisar A, Malekmohammadi M, Bronte-Stewart $\mathrm{H}$, Chizeck HJ: A metric for evaluating and comparing closed-loop deep brain stimulation algorithms. arXiv (http:// arxiv.org/abs/1605.09312) [Accessed September 20, 2016] 
8. Kuncel AM, Cooper SE, Wolgamuth BR, Clyde MA, Snyder SA, Montgomery EB Jr, et al: Clinical response to varying the stimulus parameters in deep brain stimulation for essential tremor. Mov Disord 21:1920-1928, 2006

9. Malekmohammadi M, Herron J, Velisar A, Blumenfeld Z, Trager MH, Chizeck HJ, et al: Kinematic adaptive deep brain stimulation for resting tremor in Parkinson's disease. Mov Disord 31:426-428, 2016

10. Medtronic: New Medtronic deep brain stimulation system the first to sense and record brain activity while delivering therapy. Medtronic Newsroom. August 7, 2013. (http:// newsroom.medtronic.com/phoenix.zhtml?c=251324\&p=irolnewsArticle\&id=1845602) [Accessed September 20, 2016]

11. Perlmutter JS, Mink JW: Deep brain stimulation. Annu Rev Neurosci 29:229-257, 2006

12. Pfurtscheller G, Berghold A: Patterns of cortical activation during planning of voluntary movement. Electroencephalogr Clin Neurophysiol 72:250-258, 1989

13. Ramoser H, Müller-Gerking J, Pfurtscheller G: Optimal spatial filtering of single trial EEG during imagined hand movement. IEEE Trans Rehabil Eng 8:441-446, 2000

14. Yamamoto T, Katayama Y, Ushiba J, Yoshino H, Obuchi T, Kobayashi K, et al: On-demand control system for deep brain stimulation for treatment of intention tremor. Neuromodulation 16:230-235, 2013

\section{Disclosures}

This work was supported by a donation from Medtronic. The content is solely the responsibility of the authors and does not necessarily represent the official views of Medtronic. Since submission of this article, Jeffrey A. Herron is now employed by Medtronic.

\section{Author Contributions}

Conception and design: all authors. Acquisition of data: Ko, Herron, Thompson, Brown. Analysis and interpretation of data: Ko, Herron, Thompson, Brown, Chizeck. Drafting the article: Ko, Herron, Brown, Chizeck. Critically revising the article: Ko, Herron, Thompson, Brown, Chizeck. Reviewed submitted version of manuscript: all authors. Approved the final version of the manuscript on behalf of all authors: Ko. Statistical analysis: Herron. Study supervision: Ko, Ojemann.

\section{Correspondence}

Andrew L. Ko, University of Washington, Department of Neurological Surgery, 1959 Pacific Ave. NE, Campus Box 356470, Seattle, WA 98195. email: alko00@neurosurgery.washington.edu. 